\title{
The interactions of peripheral membrane proteins with biological membranes
}

\author{
A. M. Whited ${ }^{\mathrm{a}}$ and A. Johs ${ }^{\mathrm{a}}$ \\ a Environmental Sciences Division, Oak Ridge National Laboratory, Oak Ridge, TN 37831, USA
}

Corresponding author: Alexander Johs (johsa@ornl.gov)

\begin{abstract}
The interactions of peripheral proteins with membrane surfaces are critical to many biological processes, including signaling, recognition, membrane trafficking, cell division and cell structure. On a molecular level, peripheral membrane proteins can modulate lipid composition, membrane dynamics and protein-protein interactions. Biochemical and biophysical studies have shown that these interactions are in fact highly complex, dominated by several different types of interactions, and have an interdependent effect on both the protein and membrane. Here we examine three major mechanisms underlying the interactions between peripheral membrane proteins and membranes: electrostatic interactions, hydrophobic interactions, and fatty acid modification of proteins. While experimental approaches continue to provide critical insights into specific interaction mechanisms, emerging bioinformatics resources and tools contribute to a systems-level picture of protein-lipid interactions. Through these recent advances, we begin to understand the pivotal role of protein-lipid interactions underlying complex biological functions at membrane interfaces.
\end{abstract}

Keywords: peripheral membrane proteins; membrane; lipids; electrostatic interactions; hydrophobic interactions; fatty acid modification

\section{Introduction}

The classic fluid mosaic model of the plasma membrane (Singer and Nicolson, 1972) implies a homogenous distribution of lipids in the plane of the membrane bilayer. Within 
the framework of this model, proteins interacting with the membrane have frequently been viewed as islands floating in a sea of lipids. However, since the 1990s, the complementary hypotheses of lipid domain formation and protein-mediated control of lipid packing has triggered significant interest in studying the lateral organization of biological membranes. Since that time, it has also become widely recognized that proteins play important roles in the organization of plasma membranes. Protein-lipid interactions modulate lipid composition, membrane dynamics and structure (Kozlov, 2010; Phillips et al., 2009), which are fundamentally important for numerous cellular processes ranging from cytoskeleton assembly (Cabeen and Jacobs-Wagner, 2005) and membrane trafficking to intracellular and intercellular signaling (Martin, 1998). The supramolecular assemblies emerging from these protein-lipid interactions carry out an array of specialized cellular functions. Thus, beyond the characterization of individual molecular species, understanding of the highly dynamic, yet tightly controlled, interplay between membrane-associated proteins and membrane lipids is needed to describe many higher order cellular functions.

The composition of biological membranes is complex and varies with the type of cell or cellular compartment. Changes in lipid composition can result in the formation of microdomains with distinct physical properties, which arise from the interplay between the characteristic lipid headgroups and the hydrocarbon chains (Cevc, 1993). A membrane bilayer is a dynamic environment with varying dielectric properties ranging from nonpolar within the hydrocarbon chain core to polar at the headgroup-solution interface. In addition, the interfacial headgroup region can assume significant negative charge densities, which has important implications for recognition and recruiting membrane-associated proteins. Peripheral membrane proteins are recruited to and interact with cellular membranes through a series of distinct mechanisms. Specific membranetargeting domains associate with a membrane through three major types interactions: electrostatic, hydrophobic and selective fatty acid modification. Thus, the targeted and occasionally transient interaction of a polypeptide with a bilayer is governed by a complex energy landscape.

The purpose of the present review is to summarize and exemplify mechanisms that drive the interactions between peripheral membrane proteins and membrane bilayers using 
specific examples from the literature. Furthermore, we highlight common experimental approaches to probe these interactions and bioinformatics as an emerging tool for predicting protein-membrane interactions.

We describe how charge complementarity between membrane and protein surfaces give rise to long-range electrostatic interactions, how proteins become tethered to membranes by inserting hydrophobic moieties into the hydrophobic hydrocarbon regions, and how some proteins use molecular recognition to selectively target specific membrane regions or other membrane proteins. Model membranes are widely used to probe proteinmembrane interactions and are thus the system of choice for biophysical studies. However, recent advances in experimental techniques, such as fluorescence microscopy, super-resolution microscopy and neutron scattering, have enabled studies of proteinmembrane interactions within the context of whole or live cells. Computational biology, computational resources, and a rapidly growing number of sequenced genomes and metagenomes allow us to analyze vast amounts of sequence data by identifying relevant sequence features, such as amphipathic helices or motifs for posttranslational lipid modifications. Advances in computational chemistry have enabled unprecedented insights into the interplay between peripheral membrane proteins and membranes. Although these interactions are highly complex and dynamic, there is much to be gleaned from understanding the underlying interaction mechanisms as they will help elucidate a range of cell-mediated processes, such as signaling, membrane trafficking, and cytoskeleton dynamics, but also provide insights relevant to antibiotic functionality, drug development and possible disease mechanisms.

\subsection{Lipids, bilayers and proteins}

Amphipathic lipids in aqueous solutions are entropically driven to self-assemble into bilayers, which enables compartmentalization and segregation of biochemical processes in cells. A lipid bilayer can be divided into a hydrophobic hydrocarbon core region and an interfacial, hydrophilic headgroup region. Bilayers are heterogeneous in terms of fatty acid core and headgroup composition. The membrane's interfacial region spans a 
distance of up to $15 \AA$ from the hydrophobic core to the bulk aqueous solution (White et al., 2001). The most abundant lipid species found in biological membranes are glycerophospholipids, such as phosphatidylcholine (PC), phosphatidylethanolamine (PE), phosphatidylserine (PS), phosphatidylglycerol (PG), phosphatidylinositol (PI) and phosphatidic acid (PA) (Fig. 1). Mammalian membranes also contain sphingomyelin (SM), the most common sphingolipid, and cholesterol, an amphiphilic sterol that resides between phospholipid molecules and has a hand in modulating membrane fluidity at varying concentrations depending on the tissue (Ramstedt and Slotte, 2002). SM was found to co-localize with cholesterol in plasma membranes and has been implicated in the formation of nanodomains (Mcintosh et al., 1992; Pathak and London, 2011; Smith et al., 2003).

Biomembranes are highly dynamic structures (Van Meer et al., 2008). The composition of biological membranes differs between bilayer leaflets. Individual phospholipid molecules are in a constant state of transition between the two membrane interfaces. A contingent of integral membrane proteins, broadly called flippases or translocases, shuttle the lipid molecules between leaflets, alternately enriching or depleting the concentration of a given lipid species and altering the local charge distribution (Daleke, 2007; Sharom, 2011). This lipid interchange may also lead to the existence of lipid rafts in the membrane, assemblies of specifically sequestered lipids and proteins that may play a role in trafficking and signaling. These rafts may even combine to form supramolecular structures that are observable using microscopic techniques (Brown and London, 1998; Simons and Gerl, 2010).

Fig. 1: Properties of common glycerophospholipid classes and headgroup charge characteristics. Phosphatidylcholine (PC) and phosphatidylethanolamine (PE) are both zwitterionic phospholipids and with zero net charge. Phosphatidic acid (PA), phosphatidylglycerols (PG), phosphatidylserine (PS) and phosphatidylinositol (PI), a glycolipid, are negatively charged phospholipids with a net charge of -1. Aliphatic palmitoyl chains represent the nonpolar hydrocarbon core. 


\subsection{Electrostatic interactions}

Biological membranes are typically composed of a combination of zwitterionic, PE, PC, SM and glycosphingolipids, and negatively charged phospholipids, such as PG, PS PA, PI, and cardiolipin (CL). Charged phospholipid headgroups give rise to ion concentration gradients at the membrane surface. Charged membrane surfaces give rise to long-range electrostatic interactions between peripheral proteins and lipid headgroups. Even a partially positively charged protein will be attracted to a negatively charged membrane by nonspecific electrostatic interactions. The protein's cationic amino acid residues are the main drivers in these interactions.

This type of interaction is rather nonspecific, but it successfully recruits proteins to the membrane interface based on charge complementarity. This phenomenon has important functional consequences for enzymes and transport systems that operate on charged substrates as well as peripheral proteins that bind to the membrane (Demchenko and Yesylevskyy, 2009; Mulgrew-Nesbitt et al., 2006).

However, not all peripheral proteins are cationic, and typically only one of the two membrane leaflets is negatively charged. For example, the inner membrane of E. coli cells contain predominantly zwitterionic PE localized on the cytoplasmic leaflet, while the concentration of PG is higher in the periplasmic leaflet, giving it a negative charge (Op den Kamp, 1979). This charge distribution is not uncommon in prokaryotic cell membranes although the exact lipid compositions may vary among prokaryotic species (Cevc, 1993; Ratledge and Wilkinson, 1989). In contrast, the cytoplasmic membrane interface in eukaryotic cells is enriched in anionic lipids, such as PS and PI, whereas the outer leaflet is dominated by the zwitterionic species PC, SM and glycosphingolipids, a sphingolipid with an attached carbohydrate (Thompson and Tillack, 1985). The inner mitochondrial membrane of eukaryotes contains higher proportions of CL making it negatively charged (Krebs et al., 1979). The dynamic redistribution of lipids in the membrane and the formation of membrane domains, which is in part a result of the complex phase behavior of the membranes themselves, can lead to the recruitment of proteins to the membrane surface, which in turn can trigger signaling cascades or organize other effector complexes. 
Electrostatic interactions play an important role in membrane targeting of cytoskeletal elements (Niggli, 2001), cationic toxins such as charybdotoxin (BenTal et al., 1997), other specific membrane-targeting domains (Lemmon and Ferguson, 2000), and electron carriers such as cytochrome $c$ (Rytömaa and Kinnunen, 1995; Subramanian et al., 1998; Tuominen, 2011). Cytochome $c$ is a peripheral protein active in the electron transport chain of the mitochondria that is highly conserved across species and between organisms. Data from binding assays, scaled particle theory and Van der Waals equations demonstrate the influence of membrane-bound cytochrome $c$ upon reorganization of bilayers composed of PG/PC, two lipids that intermix well without bound protein (Heimburg et al., 1999). Cytochrome $c$ has a strong preference for anionic PG lipids and its binding is enhanced upon PG redistribution and sequestration.

\subsubsection{Hydrophobic interactions}

Hydrophobic interactions are mediated by hydrophobic regions of peripheral membrane proteins. Partitioning of hydrophobic moieties into the nonpolar hydrocarbon region of the bilayer enables membrane attachment and tethering of the protein to the membrane either by insertion of a hydrophobic or amphipathic $\alpha$-helix into the membrane, discussed herein, or by a lipid anchor, such as fatty acid-modified domains, discussed in further detail in section1.2.2. These moieties may be as simple as a preponderance of exposed hydrophobic amino acid residues, which may be exposed upon receptor-induced protein unfolding (Clifton et al., 2012), or higher order structures such as amphipathic helices (Fig. 2), a common route of insertion for peptides (Dathe et al., 1996; White and Wimley, 1998), and hydrophobic loops (Gamsjaeger et al., 2005). These protein-membrane interactions frequently involve a conformational change at the membrane interface that expose a buried hydrophobic surface, which in turn facilitates insertion of a particular domain into the hydrophobic core of the membrane (Johnson and Cornell, 1999; Stahelin et al., 2003). Hydrophobic interactions are also implicated in integral protein-lipid (Grage et al., 2011; Lee, 2003) and enzyme-lipid interactions (Roberts and Rosenberry, 1985), indicating their essentiality in a range binding events. 
MinD is an ATPase that is involved in selecting the site for cellular division. While there is no clear domain responsible for its association with the membrane, the C-terminal end contains a membrane-targeting amino acid sequence that is conserved across eubacteria, archaea, and plants (Szeto et al., 2002). This domain forms an amphipathic helix, giving the hydrophobic amino acid residues access to the hydrophobic membrane interior, while the cationic charges on the opposite side of the helix interact with the anionic lipid headgroups. This surface-level attachment of MinD allows for rapid and reversible association with the membrane, enabling the protein to easily continue its functional progression along the cellular division site.

\subsubsection{Post-translational modifications}

Lipid-anchored proteins are covalently linked to different acyl chains, isoprenoids, sterols, phospholipids or glycosylphosphatidylinositol (GPI) (Paulick and Bertozzi, 2008; Resh, 2013). The obvious reason for these co- or post-translational modifications is membrane-targeting through hydrophobic interactions, but they can also facilitate protein-protein interactions and aid in the formation of membrane domains involving both peripheral and integral membrane proteins (Levental et al., 2010a). The most common post-translational modifications relevant for membrane interactions are myristoylation, prenylation, palmitoylation and the addition of a GPI anchor. Protein association with membranes through acylated residues is frequently a reversible process. For example, a 'myristoyl-conformational switch' exposes or buries the acyl chain in a hydrophobic binding pocket and thus allows reversible membrane binding of the protein (Hermida-Matsumoto and Resh, 1999). The motif MGxxxS/T may be myristoylated at the N-terminal Gly after removal of the initiating Met by methionine aminopeptidase. $\mathrm{N}$ myristoylation is catalyzed by $\mathrm{N}$-myristoyl transferase, which uses myristoyl-CoA as a substrate (Resh, 2013).

Another example of lipid modification is prenylation. It comprises the transfer of a farnesyl or geranylgeranyl group to a cysteine residue near the $\mathrm{C}$-terminus of a protein. Farnesyltransferase or geranyltransferase I recognize the motif "CaaX", where "a" corresponds to any aliphatic amino acid and " $\mathrm{X}$ " is a marker recognized by the respective 
transferase (A, C, M, Q, S = farnesyltransferase; E, L = geranyltransferase I) (Casey and Seabra, 1996; Reid et al., 2004). Palmitoylation is the covalent attachment of palmitate, a saturated fatty acid, to a cysteine by a series of enzymes (Rocks et al., 2010). In prokaryotic cells, the consensus sequence $\mathrm{LxxC}$ has been found to trigger palmitoylation. The cysteine is modified with a thioether linkage to diacylglycerol and one amide-linked fatty acid, which promotes localization to the outer membrane of Gram-negative bacteria (Hayashi and $\mathrm{Wu}, 1990$ ). This type of protein modification constitutes the basis for the assembly of many complex, membrane-associated structures in eukaryotic and bacterial cells (Linder et al., 1991). Examples include anchoring of the peptidoglycan layer to the outer membrane (Braun and Rehn, 1969), electron transport in dissimilatory reduction (Myers and Myers, 2004), immunological signaling (Levental et al., 2010b), enzymeregulated protein-protein interactions (Feron et al., 1998), ATP-binding cassette transporters (Singaraja et al., 2009), and toxins (Hackett et al., 1995).

In many cases, stable anchoring of the peripheral protein to a membrane requires more than a single covalent fatty acid modification. Typically a second feature, such as exposed hydrophobic and charged amino acids, are required for stable binding to the membrane. One such example involves the myristoylation of the Golgi reassembly stacking protein (GRASP) (Heinrich et al., 2014). Though the exact physiological role of GRASP is unknown, it is clear that N-terminal myristoylation restricts the orientation of GRASP perpendicular to the bilayer plane, consistent with trans interactions, which tether the Golgi membranes into their signature stacked structure. Without this modification, such structures are not formed as the GRASP domains form horizontal cis dimers that fail to tether the membranes together. This N-terminal-directed anchor is likely a secondary attachment site as experiments revealed that it was necessary to incorporate a His tag on the $\mathrm{C}$-terminus to bind to $\mathrm{Ni}^{2+}$-labeled lipids in order to mimic a complementary attachment to an adjacent membrane and achieve efficient tethering. Another important posttranslational fatty acid modification is the covalent attachment of a GPI anchor to the C-terminus of peripheral membrane proteins. Exclusively found in eukaryotes and a select number of archaea, these GPI-anchored proteins see the addition of saturated PI chains which in turn anchor the protein to the outer leaflet of the plasma membrane, though the biosynthesis pathways and necessary signaling sequences vary 
substantially between mammals and protozoa (Fujita and Jigami, 2008; Ikezawa, 2002). This modification plays a role in signaling, endocytosis, and intercellular interactions including adhesion and immune response (Ferguson, 1999; Ikezawa, 2002). Some studies have shown that GPI-anchored proteins may associate with lipid rafts through this fatty acid modification (Maeda et al., 2007). These rafts, residing in the membrane, are enriched in sphingolipids and cholesterol, though the native size of these floating rafts remains a matter of debate (Friedrichson and Kurzchalia, 1998; Sharma et al., 2004; Varma and Mayor, 1998). Once inserted into these rafts, the GPI-anchored proteins form cholesterol-dependent, detergent-resistant membrane (DRM) complexes (Brown, 1992; Simons and Ikonen, 1997), indicating that the fatty acid anchor is a key structural component in mediating the interactions between protein and membrane.

\subsubsection{Mechanisms for membrane-targeting}

With few exceptions, membrane targeting of peripheral membrane proteins and peptides relies on more than a single mechanism. A combination of interactions is necessary as seen in the studies on co- and posttranslational protein modifications. Two interactions that that go hand-in-hand are electrostatic and hydrophobic interactions. These types of interactions usually occur in a two-step process: 1) fast-acting, long-range electrostatic interactions draw the protein's positively charged amino acid residues to the negatively charged membrane surface, followed by 2) the slower interaction between the protein's hydrophobic amino acid residues, forming hydrophobic loops, or the induced conformation of a protein's disordered domain into an amphipathic helix (Cho and Stahelin, 2005). The partitioning of each of the 20 amino acids between the aqueous phase, the interfacial headgroup layer and the hydrocarbon core has been investigated experimentally and computationally (Wimley and White, 1996). While aliphatic side chains were shown to be involved mainly with the nonpolar core, the aromatic amino acid residues Trp and Tyr were found to exhibit an unexpectedly high affinity for the interfacial region (Gelb et al., 1999; Kachel et al., 1995).

A targeting mechanism comprised of a dual interaction membrane binding process has also been verified in equinatoxin II, a toxin derived from the sea anemone Actinia equine 
that forms pores in the membrane (Hong et al., 2002). The toxin has a strong affinity for sphingomyelin lipids. The initial attachment of the protein to the membrane is reversible and mediated mainly by two tryptophans interacting with the lipid headgroups. Following attachment to the lipid interface, the $\mathrm{N}$-terminal forms an amphipathic helix and inserts into the hydrophobic core, forming a pore that disrupts the membrane. Membrane targeting may play a pivotal role in human disease. Tau is an intrinsically disordered protein (IDP) that is involved in neurodegenerative disease. As Tau interacts with anionic lipids, its structure is altered and it becomes a compacted, partially folded protein which may be the catalyst for Tau aggregation into disease-causing neurofibrillary tangles (Jones et al., 2012). Additionally, Tau interacts hydrophobically with the core of the membrane, causing disorganization of the lipids and destabilizing the membrane structure, which may also be associated with a disease mechanism. Another protein associated with human disease is $\beta_{2}$-glycoprotein I. The protein contains a positively charged membrane-targeting domain that is responsible for rapid electrostatic binding to anionic lipid headgroups (Gamsjaeger et al., 2005) and a hydrophobic loop has been implicated in anchoring the protein to the membrane. The resulting irreversible formation of large-scale $\beta_{2}$-glycoprotein I assemblies on the membrane surface has been observed by atomic force microscopy (AFM), which is consistent with a conformational change occurring only after protein-membrane interaction which results in downstream protein-protein interactions on the membrane surface.

Some peripheral membrane protein-lipid interactions require protein modification in addition to electrostatic and hydrophobic interactions. Cellular Src kinase (c-Src), a nonreceptor tyrosine kinase, relies on myristoylation of the $\mathrm{N}$-terminus to interact hydrophobically with the membrane and the conservation of six basic amino acid residues, also located on the $\mathrm{N}$-terminal, to interact with the anionic lipid headgroups in order for membrane binding to occur (Kaplan et al., 1990; Sigal et al., 1994).

\subsection{Cytoskeletal proteins - A case study}

Bacterial cytoskeletons are ideal systems for studying peripheral protein-membrane interactions. Cytoskeletal proteins interacting with cellular membranes generally 
maintain the morphology of cells. Cytoskeletal proteins mediate diverse structural and physiological functions in bacteria from cell shape and curvature to cell division (Cabeen and Jacobs-Wagner, 2005; Carballido-López and Errington, 2003; Graumann, 2009). There are also highly conserved, interacting peripheral protein domains that electrostatically affect membrane curvature in eukaryotes (Arkhipov et al., 2008; Peter et al., 2004), indicating the universality of the morphological manifestations and the effect on lipid and protein organization of protein-lipid interactions (Kozlov, 2010; Phillips et al., 2009).

MreB, a bacterial homolog of actin, is a peripheral protein indicated in maintaining cell shape and polarity (Jones et al., 2001). MreB interacts with a ring-forming protein complex that includes MreC and MreD, both intergral membrane proteins, RodZ, and cell wall peptidogycans to give bacterial cells a rod-like shape (Vats et al., 2009). Its membrane-targeting mechanism may comprise either an $\mathrm{N}$-terminal hydrophobic loop, as identified in T. maritime, or an N-terminal amphipathic helix, as seen in E. coli (Salje et al., 2011). These direct lipid interactions may aid in bringing MreB into proximity with other proteins in the ring-forming complex, which is proceeded by the formation of this higher-order, supramolecular ring structure (Salje et al., 2011).

FtsA is also a member of the actin homolog family (van den Ent and Löwe, 2000). Like MinD discussed in section 1.2.1, it is involved in cell division. Whereas MinD is active in determining the correct location for cell division to occur, FtsA initiates the recruitment of other proteins to the divisional site, called the $\mathrm{Z}$ ring (Buddelmeijer and Beckwith, 2002), which contracts during cell division. The membrane targeting sequence on the Cterminal of FtsA is similar to the targeting sequence of MinD and it forms, like MinD, an amphipathic helix (Pichoff and Lutkenhaus, 2005). Once FtsA is bound to the membrane, it may assemble into filaments along the membrane (Szwedziak et al., 2012), allowing it recruit the crucial Z ring peripheral membrane protein FtsZ (Dai and Lutkenhaus, 1991), the tubulin-like counterpart to FtsA. Once FtsA is bound to the membrane, FtsZ binds to FtsA and forms filaments, which make up the $\mathrm{Z}$ ring, and continues the further recruitment of divisional proteins, though FtsZ and FstA alone may be capable of functional $\mathrm{Z}$ ring formation (Osawa et al., 2008). It is unclear how FtsZ causes membrane contraction at the $\mathrm{Z}$ ring during cell division, but it appears the FtsZ filaments are curved 
(Erickson, 2009; Li et al., 2007; Osawa et al., 2009) and that interaction with MreB is essential (Fenton and Gerdes, 2013).

Cellular morphology and division are complex processes that rely on numerous, wellcoordinated protein-membrane and protein coupled interactions. These interactions rely on the same interaction mechanisms reviewed herein and are often times the result of coinciding mechanisms. The large body of work on complex, bacterial cytoskeletal protein-membrane, protein-protein interactions exemplifies the importance of such interactions in all species.

\subsection{Structural bioinformatics tools for the prediction of protein-lipid interactions}

Structural genomics initiatives have generated a rapidly growing number of highresolution structures and a massive quantity of sequenced genomes. Sequence data obtained by whole genome sequencing is expected to grow exponentially for years to come (Kodama et al., 2012). Bioinformatics is a growing field with the potential to advance our understanding of structure and binding interactions through the large-scale analysis of genomic and proteomic data sets. There are many database tools available for retrieving structural, proteomic, and genomic information (PDB, GenPept, RefSeq, UniProtKB, Swiss-Prot, GenBank). A large set of sequence analysis tools is available for predicting structure and function, which can be readily applied to derive a considerable amount of useful information about peripheral membrane protein-lipid interactions. The Basic Local Alignment Search Tool (BLAST) is a widely used algorithm to compare a single query sequence with a large number of target sequences assembled in a library or database (Altschul et al., 1990; Altschul et al., 1997). It reveals sequence similarities among a large number of data sets, which is particularly useful for finding homologous or similar sequences across a broad spectrum of organisms. Multiple sequence alignment algorithms, such as Clustal Omega (Sievers et al., 2011), MAFFT (Katoh et al., 2002) and T-Coffee (Notredame et al., 2000; Tommaso et al., 2011), are typically used to identify conserved amino acid residues in a well-defined set of query sequences. In combination with existing information from proteins of known function, these 
approaches enable the identification of domains interacting with membranes based on homology or sequence similarity.

\subsubsection{Prediction of lipid modifications and signal peptides}

Proteins with membrane targeting domains may not show an overall high level of homology or sequence similarity yet may contain short, highly conserved stretches of amino acid residues. These short sequence motifs are difficult to detect with alignment algorithms, but can be quickly identified using known patterns or profiles. Certain types of posttranslational modifications, such as lipidation, are typically triggered by characteristic sequence motifs. The tool ScanProsite utilizes a user-defined pattern to identify matching motifs in a set of sequences. In addition, the PROSITE motif database can be used to identify unknown domains and functional sites in proteins (de Castro et al., 2006; Sigrist et al., 2002).

Bacterial lipoproteins comprise a large group of peripheral membrane proteins, which feature a characteristic signal sequence in the $\mathrm{N}$-terminus followed by a cysteine (Hayashi and $\mathrm{Wu}, 1990)$. This signal sequence, unlike the sequence of secreted proteins which are cleaved by signal peptidase I (SPaseI), is recognized by signal peptidase II (SPaseII). The LipoP 1.0 server predicts bacterial lipoproteins and their respective cleavage sites with 96.8\% accuracy using a hidden Markov model (HMM) trained on SPaseI-cleaved proteins, lipoproteins, and cytoplasmic and transmembrane proteins. (Juncker et al., 2003).

A similar approach is used in TMHMM 2.0 to predict transmembrane helices in proteins. TMHMM is widely used in genome annotations to discriminate membrane proteins from non-membrane proteins using genome sequencing data (Krogh et al., 2001). In addition to a high proportion of hydrophobic amino acids, an abundance of positively charged residues are associated with the cytoplasmic surface of the bacterial inner membrane proteins (von Heijne, 1992). By combining hydrophobicity analysis with charge distributions, highly accurate predictions of amphipathic and hydrophobic helices can be obtained using TMHMM.

A database of bacterial lipoproteins, DOLOP, with information for about 278 distinct lipoproteins and predicted lipoproteins from 234 completely sequenced bacterial 
genomes, has been developed (Babu et al., 2006; Babu and Sankaran, 2002). DOLOP categorizes bacterial lipoproteins based on function and provides domain assignments and links to Swiss-Prot and the PDB.

\subsubsection{Prediction of amphipathic helices}

A useful tool in determining the structure of the protein domain that is interacting with the hydrophobic core of the membrane is helical wheel analysis (Schiffer and Edmundson, 1967). To create a helical wheel, the sequence of amino acids that make up a suspected amphipathic helical region within the protein's secondary structure are plotted in a rotating manner where the angle of rotation between consecutive amino acids is $100^{\circ}$, so that the final plot represents a view down the helical axis. The plot reveals whether hydrophobic amino acids are concentrated on one side of the helix, usually with polar or hydrophilic amino acids on the other, indicating the formation of an amphipathic helix (Fig. 2). This technique can provide insight into unresolved binding mechanisms and the subsequent interactions responsible for binding (Szeto et al., 2002).

Fig. 2: Helical wheel predictions. Amino acid sequences are entered into a helical wheel prediction tool and the individual amino acids are plotted $100^{\circ}$ apart from each other, creating a helix that is 3.6 amino acids per turn. Once the amino acids have been mapped, there is either A) a preponderance of hydrophobic amino acids (yellow) on one side of the helix and amino acids containing uncharged (green) and charged (pink and blue) side chains on the other, indicating the formation of an amphipathic helix, or B) the plot shows an interspersion of hydrophobic, uncharged and charged amino acids with no clear separation and thus, no formation of an amphipathic helix.

\subsection{Experimental approaches to study protein-lipid interactions}

There are a variety of techniques available to study the different aspects of peripheral protein-membrane interactions. For a straightforward visualization of the interaction, there are several appropriate microscopic techniques, provided that the interaction results in the formation of supramolecular structures. Fluorescence microscopy (Grage et al., 2011; Heinrich et al., 2014; Jones et al., 2012; Szeto et al., 2002), AFM (Gamsjaeger et al., 2005; Grage et al., 2011), and Brewster angle microscopy (Clifton et al., 2012) have been used to successfully observe the structural and organizational effects of protein-lipid 
binding. If individual lipid molecules are tightly bound to peripheral membrane proteins, it is possible to reveal specific lipid-protein interactions at atomic resolution using $\mathrm{x}$-ray crystallography as demonstrated by the crystal structure of human N-acyl-PEhydrolyzing phospholipase D (Magotti et al., 2015). Orientations of peripheral membrane proteins of known structure relative to the membrane bilayer plane can be obtained from databases, such as the Orientations of Proteins in Membranes (OPM) database (Lomize et al., 2006).

Spectroscopic techniques can be used to determine the kinetics of a binding interaction between a peripheral membrane protein and the membrane, such as in surface plasmon resonance (SPR) (Gamsjaeger et al., 2005; Heinrich et al., 2014; Hong et al., 2002), or a restriction in mobility associated with binding, as with electron spin resonance (ESR) (Borbat et al., 2001; Gamsjaeger et al., 2005). Circular dichroism (CD) is an additional spectroscopic method that can be used to detect changes in secondary structure upon membrane binding (Latal et al., 1997; Qian et al., 2014; Szeto et al., 2002). Traditional binding assays utilizing labeled proteins can also be used effectively (Heimburg et al., 1999; Hong et al., 2002; Sigal et al., 1994).

Protein interactions can modulate the equilibrium membrane bilayer structure, but also change dynamical properties, which may be probed with several biophysical techniques. Fluorescence spectroscopic methods are among the most commonly used to study protein-membrane interactions as they offer both steady state and time-resolved capabilities. Fluorescence intensity and fluorescence quenching assays can be used to determine static parameters such as binding affinity (Lakowicz, 2007) while methods such as Förster resonance energy transfer (FRET) can be used to study dynamic interaction processes occurring within the membrane (Gorbenko and Kinnunen, 2013). Interactions between peripheral membrane proteins and membranes can result in changes of phospholipid membrane dynamics and structure, which can be probed by nuclear magnetic resonance (NMR) or electron paramagnetic resonance (EPR) spectroscopy over a range of timescales (Leftin and Brown, 2011; Malmberg and Falke, 2005; Marsh, 2010; Sanders and Landis, 1995). Membrane lipid motions and bilayer fluctuations are reflected in NMR relaxation times. EPR spectra of spin-labelled lipids are used to reveal the stoichiometry and selectivity of protein interactions for different lipid species. 
Biological and model membrane bilayers are inherently disordered as a result of a complex energy landscape. X-ray and neutron scattering provide useful insights into the structural properties of a fluid bilayer as these techniques are well suited to probe changes in the distribution of groups of molecules. Neutron scattering in combination with $\mathrm{H}_{2} \mathrm{O} / \mathrm{D}_{2} \mathrm{O}$ contrast variation and targeted isotopic labeling of membrane lipids is a unique approach for probing changes in the organization of lipid bilayer membranes induced by the interaction with peripheral membrane proteins. Deuterium labeling makes neutron techniques well-suited to study biological membranes at sub-nanometer resolution as it is non-perturbing and has a tunable sensitivity, mediated by the scattering contrast of proteins and isotopically labeled lipids. Small angle neutron scattering (SANS) and neutron reflectometry are two complementary methods for probing such interactions (Clifton et al., 2012; Grage et al., 2011; Heinrich et al., 2014; Jones et al., 2012; Lu et al., 2007; Qian et al., 2014). Neutron reflectometry is used to study the interactions at solidsupported membrane interfaces (Harroun et al., 2005) while SANS can be used to study protein interactions with membrane vesicles in solution (Kucerka et al., 2004). Recent advances in the development of accurate theoretical representations of biological membranes using molecular dynamics (MD) simulations enables interpretation of neutron scattering data and with far higher accuracy (Heberle et al., 2012; Vogel et al., 2012). MD simulations inherently account for disorder as a result of thermal fluctuations and are particularly useful for visualizing the interactions between proteins and individual lipid molecules (Demchenko and Yesylevskyy, 2009). However, MD simulations rely on the availability accurate force field parameters, which have to be refined for many classes of biological lipids. The continuous improvement of these parameters along with advances in experimental techniques will provide unprecedented insights into biological processes at membrane interfaces.

\section{Conclusions}

The binding of a peripheral membrane protein to a biological membrane can be described as a complex and cooperative process that can occur through various mechanisms. What has become clear over time is that there is a distinct interplay between the lipid membrane environment and proteins and that neither is the other's passive partner. Many 
biophysical approaches have been used to characterize protein-lipid interactions experimentally. However, only a few methods offer the ability to probe a system without perturbation by molecular labels, such as fluorescence or spin labels. Neutron reflectometry and SANS comprise label-free approaches to probe interaction-induced changes in the molecular organization of proteins and lipids in complex samples. At length scales in the nanometer to micrometer range, recent advances in microscopic techniques, such as AFM, confocal laser scanning microscopy and super-resolution microscopy have rapidly improved our ability to link molecular-scale processes to microscopic observations, such as molecular processes impacting cell shape or the formation of membrane rafts and other supramolecular structures. Computational approaches, such as molecular dynamics simulations, have become a valuable tool for understanding the complex mechanisms of protein-lipid interactions with unprecedented detail. With the rapidly growing availability of computational resources, simulations of highly complex systems over longer, biologically relevant timescales are attainable. Furthermore, the analysis of genomic information using structural bioinformatics significantly expands our ability to discover new proteins with membrane-targeting domains and to identify fundamental mechanisms common to different species and organisms. These advances will push the boundaries of our understanding about the complementary roles of proteins and membranes in cellular processes such as signaling, trafficking, and adhesion.

\section{Acknowledgments}

This research was supported by the Laboratory Directed Research and Development Program of the Oak Ridge National Laboratory (ORNL). ORNL is managed by UTBattelle, LLC under contract no. DE-AC05-00OR22725 with the US Department of Energy. The United States Government retains and the publisher, by accepting the article for publication, acknowledges that the United States Government retains a non-exclusive, paid-up, irrevocable, world-wide license to publish or reproduce the published form of this manuscript, or allow others to do so, for United States Government purposes. The 
Department of Energy will provide public access to these results of federally sponsored research in accordance with the DOE Public Access Plan (http://energy.gov/downloads/doe-public-access-plan).

\section{References}

Altschul, S.F., Gish, W., Miller, W., Myers, E.W., Lipman, D.J., 1990. Basic local alignment search tool. J Mol Biol 215, 403-410.

Altschul, S.F., Madden, T.L., Schaffer, A.A., Zhang, J., Zhang, Z., Miller, W., Lipman, D.J., 1997. Gapped BLAST and PSI-BLAST: A new generation of protein database search programs. Nucleic Acids Res 25, 3389-3402.

Arkhipov, A., Yin, Y., Schulten, K., 2008. Four-scale description of membrane sculpting by BAR domains. Biophysical journal 95, 2806-2821.

Babu, M.M., Priya, M.L., Selvan, A.T., Madera, M., Gough, J., Aravind, L., Sankaran, K., 2006. A database of bacterial lipoproteins (DOLOP) with functional assignments to predicted lipoproteins. Journal of Bacteriology 188, 2761-2773.

Babu, M.M., Sankaran, K., 2002. DOLOP-database of bacterial lipoproteins. Bioinformatics 18, 641-643.

BenTal, N., Honig, B., Miller, C., McLaughlin, S., 1997. Electrostatic binding of proteins to membranes. Theoretical predictions and experimental results with charybdotoxin and phospholipid vesicles. Biophys J 73, 1717-1727.

Borbat, P.P., Costa-Filho, A.J., Earle, K.A., Moscicki, J.K., Freed, J.H., 2001. Electron spin resonance in studies of membranes and proteins. Science 291, 266-269.

Braun, V., Rehn, K., 1969. Chemical characterization, spatial distribution and function of a lipoprotein (murein-lipoprotein) of the E. coli cell wall. European Journal of Biochemistry 10, 426-438.

Brown, D.A., 1992. Interactions between GPI-anchored proteins and membrane lipids. Trends in cell biology 2, 338-343.

Brown, D.A., London, E., 1998. Functions of lipid rafts in biological membranes. Annual Review of Cell and Developmental Biology 14, 111-136.

Buddelmeijer, N., Beckwith, J., 2002. Assembly of cell division proteins at the E. coli cell center. Current Opinion in Microbiology 5, 553-557.

Cabeen, M.T., Jacobs-Wagner, C., 2005. Bacterial cell shape. Nature Reviews Microbiology 3, 601-610.

Carballido-López, R., Errington, J., 2003. A dynamic bacterial cytoskeleton. Trends in cell biology 13, 577-583.

Casey, P.J., Seabra, M.C., 1996. Protein prenyltransferases. J Biol Chem 271, 5289-5292. Cevc, G., 1993. Phospholipids Handbook. Marcel Dekker, Inc., New York.

Cho, W.H., Stahelin, R.V., 2005. Membrane-protein interactions in cell signaling and membrane trafficking. Annu Rev Bioph Biom 34, 119-151.

Clifton, L.A., Johnson, C.L., Solovyova, A.S., Callow, P., Weiss, K.L., Ridley, H., Le Brun, A.P., Kinane, C.J., Webster, J.R.P., Holt, S.A., Lakey, J.H., 2012. Low Resolution Structure and Dynamics of a Colicin-Receptor Complex Determined by Neutron Scattering. Journal of Biological Chemistry 287, 337-346. 
Dai, K., Lutkenhaus, J., 1991. ftsZ is an essential cell division gene in Escherichia coli. Journal of bacteriology 173, 3500-3506.

Daleke, D.L., 2007. Phospholipid flippases. Journal of Biological Chemistry 282, 821825.

Dathe, M., Schümann, M., Wieprecht, T., Winkler, A., Beyermann, M., Krause, E., Matsuzaki, K., Murase, O., Bienert, M., 1996. Peptide Helicity and Membrane Surface Charge Modulate the Balance of Electrostatic and Hydrophobic Interactions with Lipid Bilayers and Biological Membranes. Biochemistry 35, 12612-12622. de Castro, E., Sigrist, C.J.A., Gattiker, A., Bulliard, V., Langendijk-Genevaux, P.S., Gasteiger, E., Bairoch, A., Hulo, N., 2006. ScanProsite: detection of PROSITE signature matches and ProRule-associated functional and structural residues in proteins. Nucleic Acids Research 34, W362-W365.

Demchenko, A.P., Yesylevskyy, S.O., 2009. Nanoscopic description of biomembrane electrostatics: results of molecular dynamics simulations and fluorescence probing. Chemistry and physics of lipids 160, 63-84.

Erickson, H.P., 2009. Modeling the physics of FtsZ assembly and force generation. Proceedings of the National Academy of Sciences 106, 9238-9243.

Fenton, A.K., Gerdes, K., 2013. Direct interaction of FtsZ and MreB is required for septum synthesis and cell division in Escherichia coli. The EMBO journal 32, 1953-1965. Ferguson, M.A., 1999. The structure, biosynthesis and functions of glycosylphosphatidylinositol anchors, and the contributions of trypanosome research. Journal of cell science 112, 2799-2809.

Feron, O., Saldana, F., Michel, J.B., Michel, T., 1998. The Endothelial Nitric-oxide Synthase-Caveolin Regulatory Cycle. Journal of Biological Chemistry 273, 3125-3128. Friedrichson, T., Kurzchalia, T.V., 1998. Microdomains of GPI-anchored proteins in living cells revealed by crosslinking. Nature 394, 802-805.

Fujita, M., Jigami, Y., 2008. Lipid remodeling of GPI-anchored proteins and its function. Biochimica et Biophysica Acta (BBA)-General Subjects 1780, 410-420.

Gamsjaeger, R., Johs, A., Gries, A., Gruber, H.J., Romanin, C., Prassl, R., Hinterdorfer, P., 2005. Membrane binding of beta2-glycoprotein I can be described by a two-state reaction model: an atomic force microscopy and surface plasmon resonance study. Biochem J 389, 665-673.

Gelb, M.H., Cho, W.H., Wilton, D.C., 1999. Interfacial binding of secreted phospholipases $\mathrm{A}(2)$ : more than electrostatics and a major pole for tryptophan. Curr Opin Struc Biol 9, 428-432.

Gorbenko, G., Kinnunen, P.K., 2013. FRET analysis of protein-lipid interactions, Fluorescent Methods to Study Biological Membranes. Springer, pp. 115-140. Grage, S.L., Keleshian, A.M., Turdzeladze, T., Battle, A.R., Tay, W.C., May, Roland P., Holt, S.A., Contera, S.A., Haertlein, M., Moulin, M., Pal, P., Rohde, P.R., Forsyth, V.T., Watts, A., Huang, K.C., Ulrich, Anne S., Martinac, B., 2011. Bilayer-Mediated Clustering and Functional Interaction of MscL Channels. Biophysical Journal 100, 12521260.

Graumann, P.L., 2009. Dynamics of bacterial cytoskeletal elements. Cell motility and the cytoskeleton 66, 909-914.

Hackett, M., Walker, C.B., Guo, L., Gray, M.C., Van Cuyk, S., Ullmann, A., Shabanowitz, J., Hunt, D.F., Hewlett, E.L., Sebo, P., 1995. Hemolytic, but Not Cell- 
invasive Activity, of Adenylate Cyclase Toxin Is Selectively Affected by Differential Fatty-acylation in Escherichia coli. Journal of Biological Chemistry 270, 20250-20253. Harroun, T.A., Fritzsche, H., Watson, M.J., Yager, K.G., Tanchak, O.M., Barrett, C.J., Katsaras, J., 2005. Variable temperature, relative humidity (0\%-100\%), and liquid neutron reflectometry sample cell suitable for polymeric and biomimetic materials. Review of Scientific Instruments 76, -. Hayashi, S., Wu, H.C., 1990. Lipoproteins in Bacteria. J Bioenerg Biomembr 22, 451471.

Heberle, F.A., Pan, J.J., Standaert, R.F., Drazba, P., Kucerka, N., Katsaras, J., 2012. Model-based approaches for the determination of lipid bilayer structure from small-angle neutron and X-ray scattering data. Eur Biophys J Biophy 41, 875-890.

Heimburg, T., Angerstein, B., Marsh, D., 1999. Binding of peripheral proteins to mixed lipid membranes: effect of lipid demixing upon binding. Biophysical journal 76, 25752586.

Heinrich, F., Nanda, H., Goh, H.Z., Bachert, C., Lösche, M., Linstedt, A.D., 2014. Myristoylation Restricts Orientation of the GRASP Domain on Membranes and Promotes Membrane Tethering. Journal of Biological Chemistry 289, 9683-9691.

Hermida-Matsumoto, L., Resh, M.D., 1999. Human immunodeficiency virus type 1 protease triggers a myristoyl switch that modulates membrane binding of Pr55(gag) and p17MA. J Virol 73, 1902-1908.

Hong, Q., Gutiérrez-Aguirre, I., Barlič, A., Malovrh, P., Kristan, K., Podlesek, Z., Maček, P., Turk, D., González-Mañas, J.M., Lakey, J.H., 2002. Two-step membrane binding by Equinatoxin II, a pore-forming toxin from the sea anemone, involves an exposed aromatic cluster and a flexible helix. Journal of Biological Chemistry 277, $41916-41924$.

Ikezawa, H., 2002. Glycosylphosphatidylinositol (GPI)-anchored proteins. Biol Pharm Bull 25, 409-417.

Johnson, J.E., Cornell, R.B., 1999. Amphitropic proteins: regulation by reversible membrane interactions (review). Molecular membrane biology 16, 217-235. Jones, E.M., Dubey, M., Camp, P.J., Vernon, B.C., Biernat, J., Mandelkow, E., Majewski, J., Chi, E.Y., 2012. Interaction of Tau Protein with Model Lipid Membranes Induces Tau Structural Compaction and Membrane Disruption. Biochemistry 51, 25392550.

Jones, L.J.F., Carballido-López, R., Errington, J., 2001. Control of Cell Shape in Bacteria: Helical, Actin-like Filaments in Bacillus subtilis. Cell 104, 913-922. Juncker, A.S., Willenbrock, H., Von Heijne, G., Brunak, S., Nielsen, H., Krogh, A., 2003. Prediction of lipoprotein signal peptides in Gram-negative bacteria. Protein Sci 12, 1652-1662.

Kachel, K., Asuncionpunzalan, E., London, E., 1995. Anchoring of Tryptophan and Tyrosine Analogs at the Hydrocarbon Polar Boundary in Model Membrane-Vesicles Parallax Analysis of Fluorescence Quenching Induced by Nitroxide-Labeled Phospholipids. Biochemistry 34, 15475-15479.

Kaplan, J.M., Varmus, H.E., Bishop, J.M., 1990. The src protein contains multiple domains for specific attachment to membranes. Molecular and Cellular Biology 10, 1000-1009. 
Katoh, K., Misawa, K., Kuma, K., Miyata, T., 2002. MAFFT: a novel method for rapid multiple sequence alignment based on fast Fourier transform. Nucleic Acids Research 30, 3059-3066.

Kodama, Y., Shumway, M., Leinonen, R., 2012. The sequence read archive: explosive growth of sequencing data. Nucleic Acids Research 40, D54-D56.

Kozlov, M.M., 2010. Biophysics: Joint effort bends membrane. Nature 463, 439-440.

Krebs, J.J.R., Hauser, H., Carafoli, E., 1979. Asymmetric Distribution of Phospholipids in the Inner Membrane of Beef-Heart Mitochondria. J Biol Chem 254, 5308-5316.

Krogh, A., Larsson, B., von Heijne, G., Sonnhammer, E.L., 2001. Predicting transmembrane protein topology with a hidden Markov model: application to complete genomes. J Mol Biol 305, 567-580.

Kucerka, N., Nagle, J.F., Feller, S.E., Balgavy, P., 2004. Models to analyze small-angle neutron scattering from unilamellar lipid vesicles. Phys Rev E Stat Nonlin Soft Matter Phys 69, 051903.

Lakowicz, J.R., 2007. Principles of fluorescence spectroscopy. Springer Science \& Business Media.

Latal, A., Degovics, G., Epand, R.F., Epand, R.M., Lohner, K., 1997. Structural Aspects of the Interaction of peptidyl-glycylleucine-carboxyamide, a Highly Potent Antimicrobial Peptide from Frog Skin, with Lipids. European Journal of Biochemistry 248, 938-946. Lee, A., 2003. Lipid-protein interactions in biological membranes: a structural perspective. Biochimica et Biophysica Acta (BBA)-Biomembranes 1612, 1-40. Leftin, A., Brown, M.F., 2011. An NMR database for simulations of membrane dynamics. Bba-Biomembranes 1808, 818-839.

Lemmon, M.A., Ferguson, K.M., 2000. Signal-dependent membrane targeting by pleckstrin homology $(\mathrm{PH})$ domains. Biochem J 350, 1-18.

Levental, I., Grzybek, M., Simons, K., 2010a. Greasing Their Way: Lipid Modifications Determine Protein Association with Membrane Rafts. Biochemistry 49, 6305-6316.

Levental, I., Lingwood, D., Grzybek, M., Coskun, Ü., Simons, K., 2010b. Palmitoylation regulates raft affinity for the majority of integral raft proteins. Proceedings of the National Academy of Sciences 107, 22050-22054.

Li, Z., Trimble, M.J., Brun, Y.V., Jensen, G.J., 2007. The structure of FtsZ filaments in vivo suggests a force-generating role in cell division. The EMBO journal 26, 4694-4708. Linder, M.E., Pang, I.H., Duronio, R.J., Gordon, J.I., Sternweis, P.C., Gilman, A.G., 1991. Lipid modifications of $G$ protein subunits. Myristoylation of Go alpha increases its affinity for beta gamma. Journal of Biological Chemistry 266, 4654-4659.

Lomize, M.A., Lomize, A.L., Pogozheva, I.D., Mosberg, H.I., 2006. OPM: Orientations of Proteins in Membranes database. Bioinformatics 22, 623-625.

Lu, J.R., Zhao, X., Yaseen, M., 2007. Protein adsorption studied by neutron reflection. Current Opinion in Colloid \& Interface Science 12, 9-16.

Maeda, Y., Tashima, Y., Houjou, T., Fujita, M., Yoko-o, T., Jigami, Y., Taguchi, R., Kinoshita, T., 2007. Fatty acid remodeling of GPI-anchored proteins is required for their raft association. Molecular biology of the cell 18, 1497-1506.

Magotti, P., Bauer, I., Igarashi, M., Babagoli, M., Marotta, R., Piomelli, D., Garau, G., 2015. Structure of Human N-Acylphosphatidylethanolamine-Hydrolyzing Phospholipase D: Regulation of Fatty Acid Ethanolamide Biosynthesis by Bile Acids. Structure 23, 598604. 
Malmberg, N.J., Falke, J.J., 2005. Use of EPR power saturation to analyze the membrane-docking geometries of peripheral proteins: applications to $\mathrm{C} 2$ domains. Annual review of biophysics and biomolecular structure 34, 71 .

Marsh, D., 2010. Electron spin resonance in membrane research: protein-lipid interactions from challenging beginnings to state of the art. European biophysics journal : EBJ 39, 513-525.

Martin, T.F.J., 1998. Phosphoinositide lipids as signaling molecules: Common themes for signal transduction, cytoskeletal regulation, and membrane trafficking. Annual Review of Cell and Developmental Biology 14, 231-264.

Mcintosh, T.J., Simon, S.A., Needham, D., Huang, C.H., 1992. Structure and Cohesive Properties of Sphingomyelin Cholesterol Bilayers. Biochemistry 31, 2012-2020.

Mulgrew-Nesbitt, A., Diraviyam, K., Wang, J., Singh, S., Murray, P., Li, Z., Rogers, L., Mirkovic, N., Murray, D., 2006. The role of electrostatics in protein-membrane interactions. Biochimica et Biophysica Acta (BBA)-Molecular and Cell Biology of Lipids 1761, 812-826.

Myers, C.R., Myers, J.M., 2004. The outer membrane cytochromes of Shewanella oneidensis MR-1 are lipoproteins. Lett Appl Microbiol 39, 466-470.

Niggli, V., 2001. Structural properties of lipid-binding sites in cytoskeletal proteins. Trends Biochem Sci 26, 604-611.

Notredame, C., Higgins, D.G., Heringa, J., 2000. T-Coffee: A novel method for fast and accurate multiple sequence alignment. J Mol Biol 302, 205-217.

Op den Kamp, J.A.F., 1979. Lipid Asymmetry in Membranes. Annual Review of Biochemistry 48, 47-71.

Osawa, M., Anderson, D.E., Erickson, H.P., 2008. Reconstitution of contractile FtsZ rings in liposomes. Science 320, 792-794.

Osawa, M., Anderson, D.E., Erickson, H.P., 2009. Curved FtsZ protofilaments generate bending forces on liposome membranes. The EMBO journal 28, 3476-3484.

Pathak, P., London, E., 2011. Measurement of Lipid Nanodomain (Raft) Formation and Size in Sphingomyelin/POPC/Cholesterol Vesicles Shows TX-100 and Transmembrane Helices Increase Domain Size by Coalescing Preexisting Nanodomains But Do Not Induce Domain Formation. Biophys J 101, 2417-2425.

Paulick, M.G., Bertozzi, C.R., 2008. The glycosylphosphatidylinositol anchor: A complex membrane-anchoring structure for proteins. Biochemistry 47, 6991-7000. Peter, B.J., Kent, H.M., Mills, I.G., Vallis, Y., Butler, P.J.G., Evans, P.R., McMahon, H.T., 2004. BAR Domains as Sensors of Membrane Curvature: The Amphiphysin BAR Structure. Science 303, 495-499.

Phillips, R., Ursell, T., Wiggins, P., Sens, P., 2009. Emerging roles for lipids in shaping membrane-protein function. Nature 459, 379-385.

Pichoff, S., Lutkenhaus, J., 2005. Tethering the Z ring to the membrane through a conserved membrane targeting sequence in FtsA. Molecular microbiology 55, 17221734.

Qian, S., Rai, D., Heller, W.T., 2014. Alamethicin Disrupts the Cholesterol Distribution in Dimyristoyl Phosphatidylcholine-Cholesterol Lipid Bilayers. The Journal of Physical Chemistry B 118, 11200-11208.

Ramstedt, B., Slotte, J.P., 2002. Membrane properties of sphingomyelins. Febs Lett 531, 33-37. 
Ratledge, C., Wilkinson, S.G., 1989. Microbial Lipids. Academic Press, London.

Reid, T.S., Terry, K.L., Casey, P.J., Beese, L.S., 2004. Crystallographic analysis of CaaX prenyltransferases complexed with substrates defines rules of protein substrate selectivity. J Mol Biol 343, 417-433.

Resh, M.D., 2013. Covalent lipid modifications of proteins. Curr Biol 23, R431-R435.

Roberts, W.L., Rosenberry, T.L., 1985. Identification of covalently attached fatty acids in the hydrophobic membrane-binding domain of human erythrocyte acetylcholinesterase. Biochemical and Biophysical Research Communications 133, 621-627.

Rocks, O., Gerauer, M., Vartak, N., Koch, S., Huang, Z.P., Pechlivanis, M., Kuhlmann, J., Brunsveld, L., Chandra, A., Ellinger, B., Waldmann, H., Bastiaens, P.I.H., 2010. The Palmitoylation Machinery Is a Spatially Organizing System for Peripheral Membrane Proteins. Cell 141, 458-471.

Rytömaa, M., Kinnunen, P.K., 1995. Reversibility of the Binding of Cytochrome c to Liposomes. Implications for lipid-protein interactions. Journal of Biological Chemistry 270, 3197-3202.

Salje, J., van den Ent, F., de Boer, P., Löwe, J., 2011. Direct membrane binding by bacterial actin MreB. Molecular cell 43, 478-487.

Sanders, C.R., Landis, G.C., 1995. Reconstitution of Membrane Proteins into Lipid-Rich Bilayered Mixed Micelles for NMR Studies. Biochemistry 34, 4030-4040.

Schiffer, M., Edmundson, A.B., 1967. Use of Helical Wheels to Represent the Structures of Proteins and to Identify Segments with Helical Potential. Biophysical Journal 7, 121135.

Sharma, P., Varma, R., Sarasij, R.C., Ira, Gousset, K., Krishnamoorthy, G., Rao, M., Mayor, S., 2004. Nanoscale Organization of Multiple GPI-Anchored Proteins in Living Cell Membranes. Cell 116, 577-589.

Sharom, F.J., 2011. Flipping and flopping-lipids on the move. IUBMB Life 63, 736746.

Sievers, F., Wilm, A., Dineen, D., Gibson, T.J., Karplus, K., Li, W.Z., Lopez, R., McWilliam, H., Remmert, M., Soding, J., Thompson, J.D., Higgins, D.G., 2011. Fast, scalable generation of high-quality protein multiple sequence alignments using Clustal Omega. Mol Syst Biol 7, 539.

Sigal, C.T., Zhou, W., Buser, C.A., McLaughlin, S., Resh, M.D., 1994. Amino-terminal basic residues of Src mediate membrane binding through electrostatic interaction with acidic phospholipids. Proceedings of the National Academy of Sciences 91, 1225312257.

Sigrist, C.J., Cerutti, L., Hulo, N., Gattiker, A., Falquet, L., Pagni, M., Bairoch, A., Bucher, P., 2002. PROSITE: a documented database using patterns and profiles as motif descriptors. Brief Bioinform 3, 265-274.

Simons, K., Gerl, M.J., 2010. Revitalizing membrane rafts: new tools and insights. Nature reviews Molecular cell biology 11, 688-699.

Simons, K., Ikonen, E., 1997. Functional rafts in cell membranes. Nature 387, 569-572. Singaraja, R.R., Kang, M.H., Vaid, K., Sanders, S.S., Vilas, G.L., Arstikaitis, P., Coutinho, J., Drisdel, R.C., El-Husseini, A.E.D., Green, W.N., Berthiaume, L., Hayden, M.R., 2009. Palmitoylation of ATP-Binding Cassette Transporter A1 Is Essential for Its Trafficking and Function. Circulation Research 105, 138-147. 
Singer, S.J., Nicolson, G.L., 1972. Fluid Mosaic Model of Structure of Cell-Membranes. Science 175, 720-\&.

Smith, A.K., Buboltz, J., Spink, C.H., Feigenson, G.W., 2003. Ternary phase diagram of the lipid mixture sphingomyelin/DOPC/cholesterol. Biophys J 84, 372a-372a.

Stahelin, R.V., Long, F., Peter, B.J., Murray, D., De Camilli, P., McMahon, H.T., Cho, W., 2003. Contrasting Membrane Interaction Mechanisms of AP180 N-terminal Homology (ANTH) and Epsin N-terminal Homology (ENTH) Domains. Journal of Biological Chemistry 278, 28993-28999.

Subramanian, M., Jutila, A., Kinnunen, P.K., 1998. Binding and dissociation of cytochrome $\mathrm{c}$ to and from membranes containing acidic phospholipids. Biochemistry 37 , 1394-1402.

Szeto, T.H., Rowland, S.L., Rothfield, L.I., King, G.F., 2002. Membrane localization of MinD is mediated by a C-terminal motif that is conserved across eubacteria, archaea, and chloroplasts. Proceedings of the National Academy of Sciences 99, 15693-15698.

Szwedziak, P., Wang, Q., Freund, S., Löwe, J., 2012. FtsA forms actin-like protofilaments. The EMBO journal 31, 2249-2260.

Thompson, T.E., Tillack, T.W., 1985. Organization of Glycosphingolipids in Bilayers and Plasma-Membranes of Mammalian-Cells. Annu Rev Biophys Bio 14, 361-386.

Tommaso, P., Moretti, S., Xenarios, I., Orobitg, M., Montanyola, A., Chang, J.M., Taly, J.F., Notredame, C., 2011. T-Coffee: a web server for the multiple sequence alignment of protein and RNA sequences using structural information and homology extension.

Nucleic Acids Research 39, W13-W17.

Tuominen, E., 2011. Phospholipid cytochrome c interactions.

van den Ent, F., Löwe, J., 2000. Crystal structure of the cell division protein FtsA from Thermotoga maritima. The EMBO journal 19, 5300-5307.

Van Meer, G., Voelker, D.R., Feigenson, G.W., 2008. Membrane lipids: where they are and how they behave. Nature reviews molecular cell biology 9, 112-124.

Varma, R., Mayor, S., 1998. GPI-anchored proteins are organized in submicron domains at the cell surface. Nature 394, 798-801.

Vats, P., Shih, Y.L., Rothfield, L., 2009. Assembly of the MreB-associated cytoskeletal ring of Escherichia coli. Molecular microbiology 72, 170-182.

Vogel, A., Roark, M., Feller, S.E., 2012. A reinterpretation of neutron scattering experiments on a lipidated Ras peptide using replica exchange molecular dynamics. BbaBiomembranes 1818, 219-224.

von Heijne, G., 1992. Membrane-Protein Structure Prediction - Hydrophobicity Analysis and the Positive-inside Rule. J Mol Biol 225, 487-494.

White, S.H., Ladokhin, A.S., Jayasinghe, S., Hristova, K., 2001. How membranes shape protein structure. J Biol Chem 276, 32395-32398.

White, S.H., Wimley, W.C., 1998. Hydrophobic interactions of peptides with membrane interfaces. Biochimica et Biophysica Acta (BBA)-Reviews on Biomembranes 1376, 339352.

Wimley, W.C., White, S.H., 1996. Experimentally determined hydrophobicity scale for proteins at membrane interfaces. Nat Struct Biol 3, 842-848. 


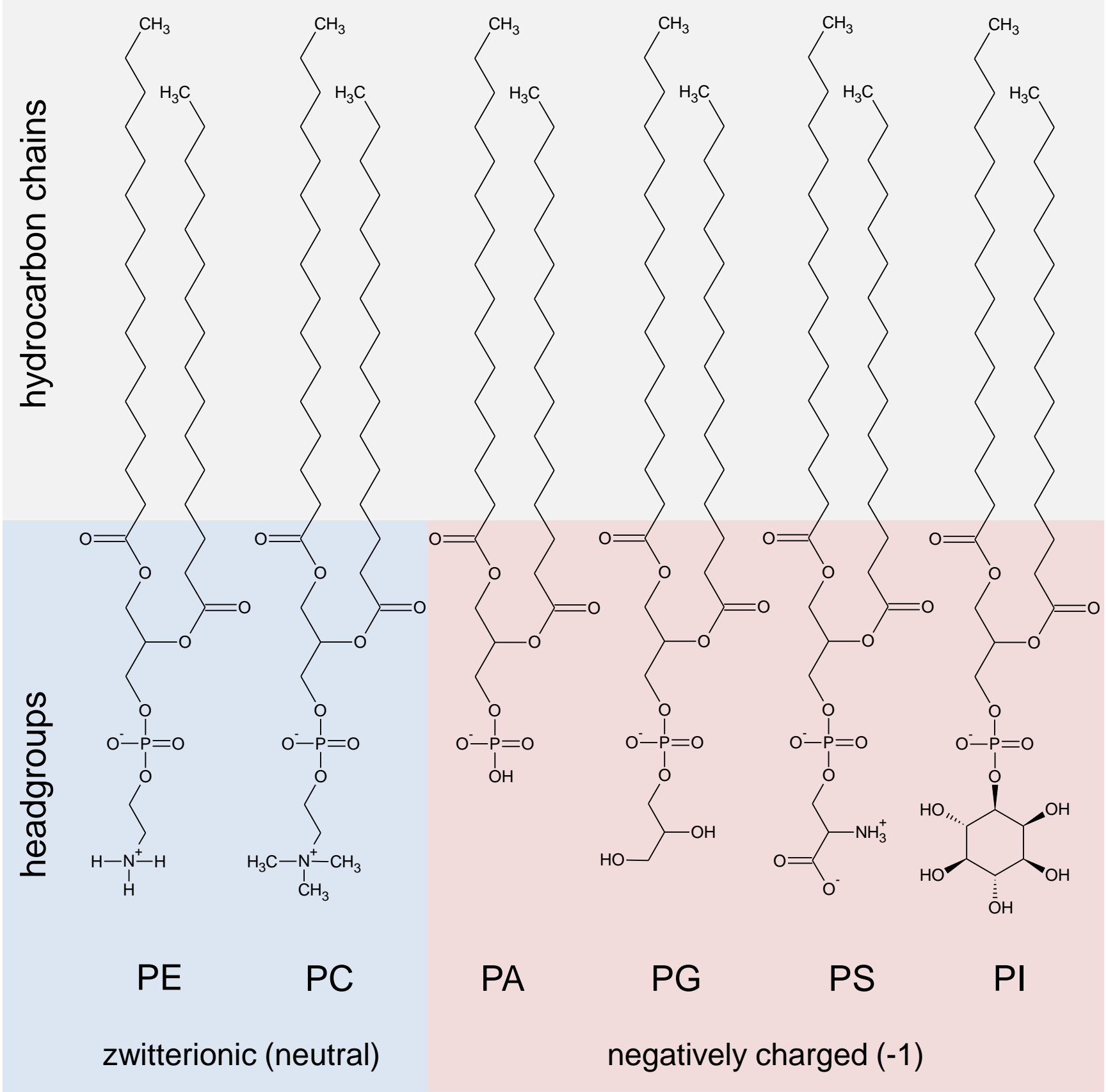


A

GLLDKVRGWMA

1

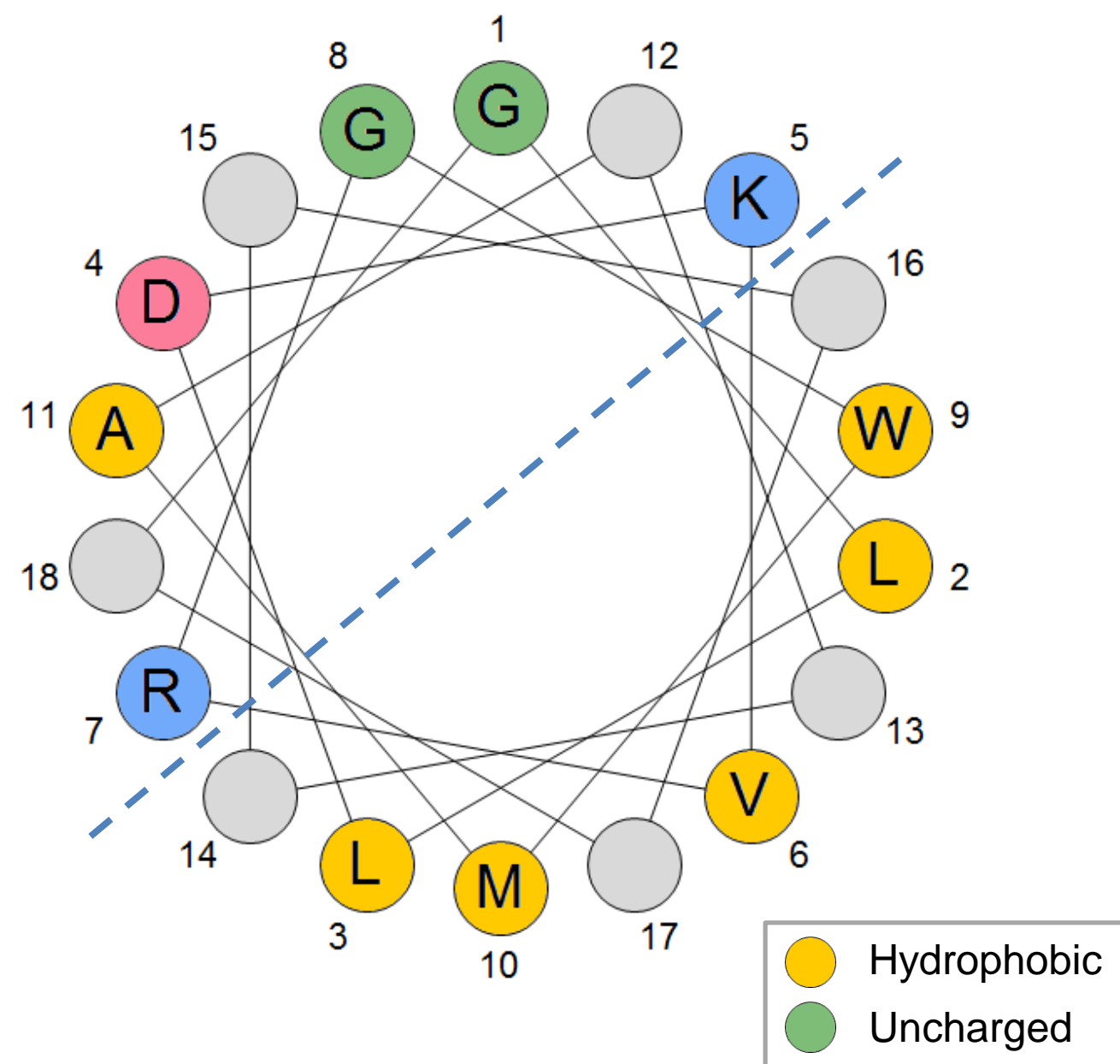

B

\section{EKHSGNEPSVT}

1

11

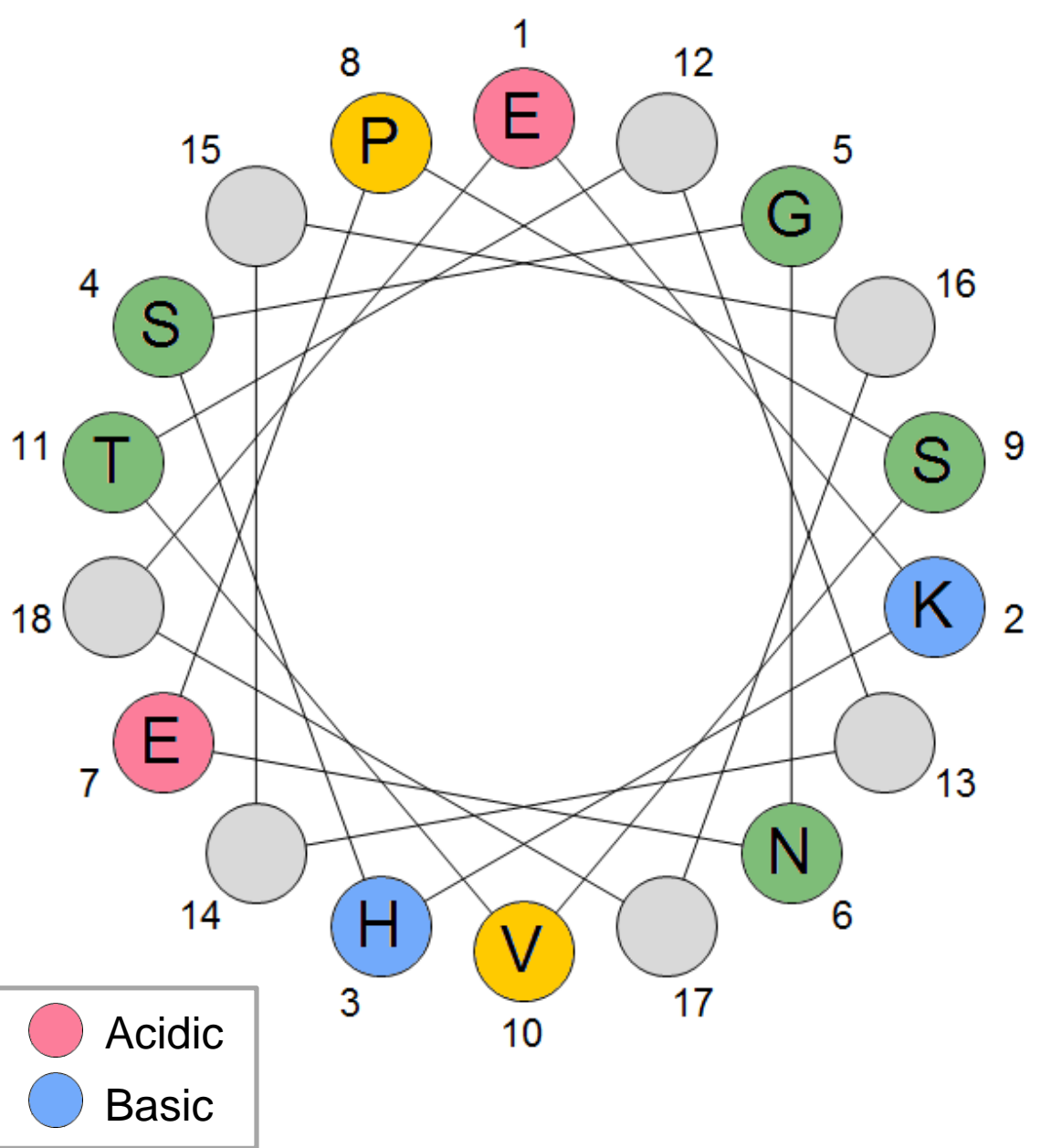

